\title{
An Interface Circuit for High-Accuracy Signal Processing of Differential-Capacitance Transducers
}

\author{
Kouji Mochizuki, Member, IEEE, Takashi Masuda, and Kenzo Watanabe, Fellow, IEEE
}

\begin{abstract}
An interface circuitry for high-accuracy signal processing of differential-capacitance transducers is developed. The architecture is based on the idea that the ratio of one of the transducer capacitances to its total capacitance represents the offset binary equivalent of the physical quantity under measurement. An opamp-based capacitance-to-voltage converter is commonly used for capacitance detection, and an analog-to-digital (A/D) converter is used for the ratiometric operation. Analyses show that the interface can detect the capacitance change as small as $0.01 \%$ of the total capacitance. Experimental results are also given to confirm the analyses.
\end{abstract}

Index Terms-Analog circuit, capacitance transducers, intelligent sensors, pressure measurement, signal processing.

\section{INTRODUCTION}

D IFFERENTIAL-CAPACITANCE transducers are widely used for detecting such physical quantities as pressure difference, linear displacement, and rotational angles. Their configurations depend on what quantity is to be measured [1], [2], but they can be represented by two capacitors whose capacitances change complementarily with the measurand. The complementary capacitance change may be linear or nonlinear. In either case, the accurate and linear detection of the measurand can be made by ratiometric signal processing which divides the capacitance difference between two capacitors by their sum. Beside such a ratiometric processing, a differential-capacitance transducer requires a special interface configuration because the two capacitors share one electrode in structure.

To meet these requirements, several techniques have so far been proposed, including switched-capacitor (SC), analog-todigital (A/D) [3], [4], capacitance-to-frequency (C/F) [5], [6], and capacitance-to-phase conversion [7]. The SC technique is best suited for integrated circuit realization using CMOS process, but suffers seriously from clock feedthrough. Because of this error source, the resolution attainable with the $\mathrm{SC}$ method is estimated to be $1 \mathrm{fF}$ [8]. A higher resolution is possible with the $\mathrm{C} / \mathrm{F}$ conversion techniques [6], but their applications to high-speed real-time measurements seem difficult because they require a microprocessor for the calibration and the ratiometric operation.

Manuscript received July 12, 1996; revised December 10, 1998.

K. Mochizuki is with the Department of Electrical Engineering, Numazu College of Technology, Numazu 410-8501, Japan.

T. Masuda is with the Engineering Department, Yamatake-Honeywell Co. Ltd., Fujisawa 251-0015, Japan.

K. Watanabe is with the Research Institute of Electronics, Shizuoka University, Hamamatsu 432-8011, Japan.

Publisher Item Identifier S 0018-9456(98)09902-1.

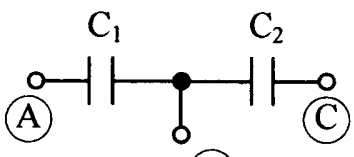

(B)

Fig. 1. An equivalent circuit of a differential-capacitance transducer.

The above-mentioned methods detect the capacitances of the transducer by sensing the charge stored in it. Sensing the current flowing through the transducer is another approach. Recent advances in high-speed opamps have made this approach quite attractive because they allow high-sensitivity and highspeed capacitance detection by means of the high-frequency probe signal. This paper describes an interface circuitry of a differential-pressure transducer based on this approach and demonstrates its performances.

\section{TRANSDUCER MODEL}

A differential-capacitance transducer can be represented by two capacitors with a common electrode, as shown in Fig. 1. In a linear displacement encoder, the areas of the electrodes change linearly with the displacement $x$. In a rotational angle encoder, the apparent permittivities of the capacitors change linearly with the angle $x$. In these encoders, therefore, the capacitances $C_{1}$ and $C_{2}$ change linearly with the measurand $x$ and can be expressed as follows:

$$
\begin{aligned}
& C_{1}=\frac{C_{0}}{2}(1+x) \\
& C_{2}=\frac{C_{0}}{2}(1-x)
\end{aligned}
$$

where $C_{0}$ is the total capacitance of the transducer. In a differential-pressure transducer, on the other hand, the spacings between the electrodes change linearly with the pressure difference $x$. Therefore, $C_{1}$ and $C_{2}$ are expressed as

$$
\begin{aligned}
& C_{1}=\frac{C_{0}}{2} \frac{1}{1-x} \\
& C_{2}=\frac{C_{0}}{2} \frac{1}{1+x} .
\end{aligned}
$$

Whether the capacitance change is linear or hyperbolic, the measurand $x$ can be detected independently of the total capacitance $C_{0}$ by the following ratiometric operation:

$$
\frac{C_{1}-C_{2}}{C_{1}+C_{2}}=x .
$$




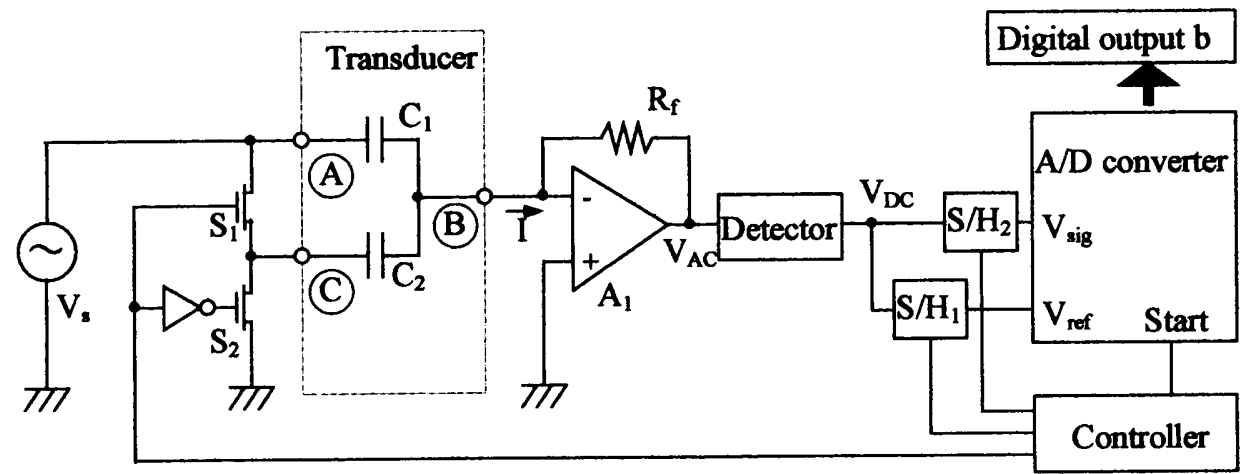

Fig. 2. A block diagram of the interface.

\section{INTERFACE CIRCUITRY}

A block diagram of the interface circuit to perform the ratiometric operation (5) is shown in Fig. 2. The circuit is designed based on the idea that component errors do not affect the ratiometric operation if a common stage is used for detecting $C_{1}-C_{2}$ and $C_{1}+C_{2}$.

The first stage is the capacitance-to-voltage converter. Sensing the current flowing through the transducer, it produces the output voltage proportional to the transducer capacitance. Assume for the moment that opamp $A_{1}$ and switches $S_{1}$ and $S_{2}$ are ideal. When $S_{1}$ is on and $S_{2}$ is off, the C/F converter senses the currents flowing through $C_{1}$ and $C_{2}$ to produce the output voltage $V_{o 1}$

$$
V_{o 1}=-s\left(C_{1}+C_{2}\right) R_{f} V_{s}
$$

where $V_{s}$ is the probe signal applied to the transducer. After conversion to a dc signal by the detector, this voltage is applied to the A/D converter as the reference voltage. When $S_{1}$ is off and $S_{2}$ is on, on the other hand, the C/F converter senses only the current flowing through $C_{1}$ to produce the output voltage $V_{o 2}$

$$
V_{o 2}=-s C_{1} R_{f} V_{s}
$$

After being converted to a dc signal by the detector, $V_{o 2}$ is applied to the signal input terminal of the $A / D$ converter. The $n$-bit $\mathrm{A} / \mathrm{D}$ converter then generates the binary number $b$ given by

$b=\frac{V_{o 2}}{V_{o 1}}=\frac{C_{1}}{C_{1}+C_{2}}=b_{1} 2^{-1}+b_{2} 2^{-2}+\cdots+b_{n} 2^{-n}$.

Modifying (5) as

$$
x=\frac{C_{1}-C_{2}}{C_{1}+C_{2}}=\frac{2 C_{1}}{C_{1}+C_{2}}-1
$$

and substituting (8) into (9), one finds that $b$ is itself the $n$-bit offset binary representation of $x$.

The circuit diagram of the detector is shown in Fig. 3. It consists of a full-wave rectifier followed by a low-pass filter [9]. The low-pass output contains the ripple component. This component should be made small enough so that the ripple factor, defined as the ratio of the ripple component to the average dc output, is smaller than the relative measurement

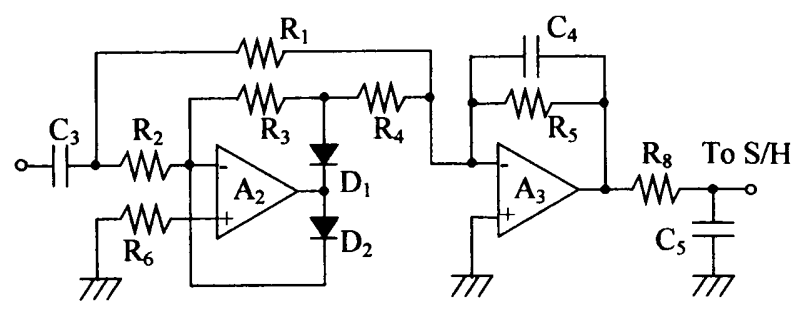

Fig. 3. The circuit diagram of the detector.

error $\varepsilon$ specified for $x$. This requirement specifies the time constant, $\tau_{C}=C_{4} R_{5}$, of the low-pass filter

$$
\tau_{C} \geq \frac{1}{2 f \ln (1-\varepsilon)}
$$

where $f$ is the frequency of the sinusoidal source $V_{s}$ applied to the transducer. The $\varepsilon \%$ settling time of the low-pass filter is then given by

$$
\tau_{S}=-\tau_{C} \ln \varepsilon
$$

The settling time $\tau_{S}$ specifies the sampling speed of the $\mathrm{S} / \mathrm{H}$ circuit, thereby limiting the conversion rate of the A/D converter to $f \ln (1-\varepsilon) / \ln \varepsilon$. Specifically, when $f=1 \mathrm{MHz}$ and $\varepsilon=0.1 \%$, the conversion rate is 150 samples per second (sps).

The above estimate of the sampling speed assuming the firstorder low-pass filter is pessimistic. The output of the detector settles much faster because $V_{o 1}$ and $V_{o 2}$ change slowly. In addition, the time constant $\tau_{C}$ can be made much smaller by adopting a second-order low-pass filter. Therefore, much higher speed can be expected without degrading the accuracy.

\section{ACCURACY}

Error sources involved in the interface circuit are parasitic capacitances, the input impedance and finite gain of opamp $\mathrm{A}$, and the nonlinearity of the detector. Their effects on the accuracy are evaluated in this section.

The $\mathrm{C} / \mathrm{V}$ converter involving the error sources is shown in Fig. 4. Exact analyses give the following expressions for the output voltages:

$$
V_{o(1,2)}^{\prime}=-\frac{Y_{s(1,2)}}{Y_{f}} \cdot \frac{k_{(1,2)}}{1+\frac{1}{A}\left(1+\frac{Y_{i}+Y_{s(1,2)}}{Y_{f}}\right)} V_{s}
$$




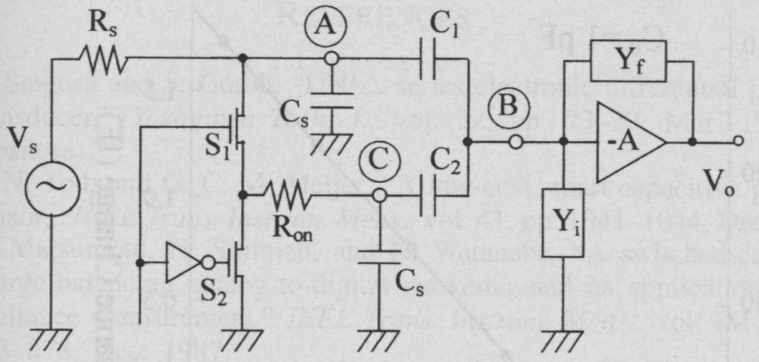

Fig. 4. The $\mathrm{C} / \mathrm{V}$ converter including error sources.

TABLE I

ERRORS DUE TO PARASITICS

\begin{tabular}{ccccccc}
$f$ & $\mathrm{R}_{\mathrm{m}}$ & $\mathrm{C}_{\mathrm{s}}$ & $\mathrm{C}_{0}$ & \multicolumn{3}{c}{ Enor $\varepsilon\left(\times 10^{4}\right)$} \\
{$[\mathrm{MHz}]$} & {$[\mathrm{W}]$} & {$[\mathrm{pF}]$} & {$[\mathrm{pF}]$} & $\mathrm{x}=-0.5$ & $\mathrm{x}=0$ & $\mathrm{x}=0.5$ \\
\hline 1 & 300 & 10 & 6 & 4.26 & 2.25 & 1.12 \\
0.5 & 300 & 10 & 6 & 1.07 & 0.56 & 0.28 \\
1 & 125 & 10 & 6 & 0.74 & 0.39 & 0.19 \\
1 & 300 & 5 & 1 & 0.60 & 0.40 & 0.22 \\
\hline
\end{tabular}

where $V_{o 1}^{\prime}$ and $V_{o 2}^{\prime}$ are the output voltages of opamp $A_{1}$ in the state 1 when $S_{1}$ is on and $S_{2}$ is off and in the state 2 when $S_{1}$ is off and $S_{2}$ is on, respectively. $A$ and $Y_{i}$ are the open-loop gain and input admittance of the opamp, respectively. $Y_{s 1}$ and $Y_{s 2}$ are the admittances of the transducer network in the state 1 and 2, respectively, seen from the inverting input terminal of opamp, and are given by

$$
\begin{aligned}
& Y_{s 1}=\left(s C_{1}+\frac{1}{R_{\text {on }}+1 / s C_{2}}\right) \| \frac{1}{R_{s}} \\
& Y_{s 2}=\frac{1}{R_{s}+1 / s C_{1}}+\frac{1}{R_{\text {on }}+1 / s C_{2}} .
\end{aligned}
$$

$Y_{f}$ is the admittance of the feedback network and $k(1,2)$ are given by

$$
\begin{aligned}
& k_{1}=\frac{1+s \frac{C_{1}\left(C_{s}+C_{2}\right)}{C_{1}+C_{2}} R_{\mathrm{on}}}{1+s\left(C_{s}+\frac{C_{1} C_{2}}{C_{1}+C_{2}}\right) R_{\mathrm{on}}} \\
& k_{2}=\frac{C_{1}}{C_{1}+C_{2}} \frac{1+s\left(C_{s}+C_{2}\right) R_{\mathrm{on}}}{1+s\left(C_{s}+\frac{C_{1} C_{2}}{C_{1}+C_{2}}\right) R_{\mathrm{on}}} .
\end{aligned}
$$

When $\omega C_{1} R_{s} \ll 1$, which is a reasonable condition in practice, $Y_{s 1}=Y_{s 2}$ holds. Then, $A$ and $Y_{i}$ have no effect on the ratiometric operation and the $\mathrm{A} / \mathrm{D}$ converter outputs the digital equivalent of $\left|k_{2}\right| /\left|k_{1}\right|$

$$
\begin{aligned}
\frac{\left|k_{2}\right|}{\left|k_{1}\right|} & =\frac{C_{1}}{C_{1}+C_{2}} \frac{\left|1+s\left(C_{s}+C_{2}\right) R_{\mathrm{on}}\right|}{\left|1+s \frac{C_{1}\left(C_{s}+C_{2}\right)}{C_{1}+C_{2}} R_{\mathrm{on}}\right|} \\
& =\frac{C_{1}}{C_{1}+C_{2}}(1+\varepsilon)
\end{aligned}
$$

where $\varepsilon$ is the relative error given, to first order, by

$$
\varepsilon=\frac{1}{2} \omega^{2}\left(C_{s}+C_{2}\right)^{2} \frac{\left(2 C_{1}+C_{2}\right) \cdot C_{2}}{C_{0}^{2}} R_{\mathrm{on}}^{2}
$$

Table I lists the error $\varepsilon$ evaluated for several cases. $R_{\text {on }}$ and $C_{s}$ quoted in this table assume standard analog switches and

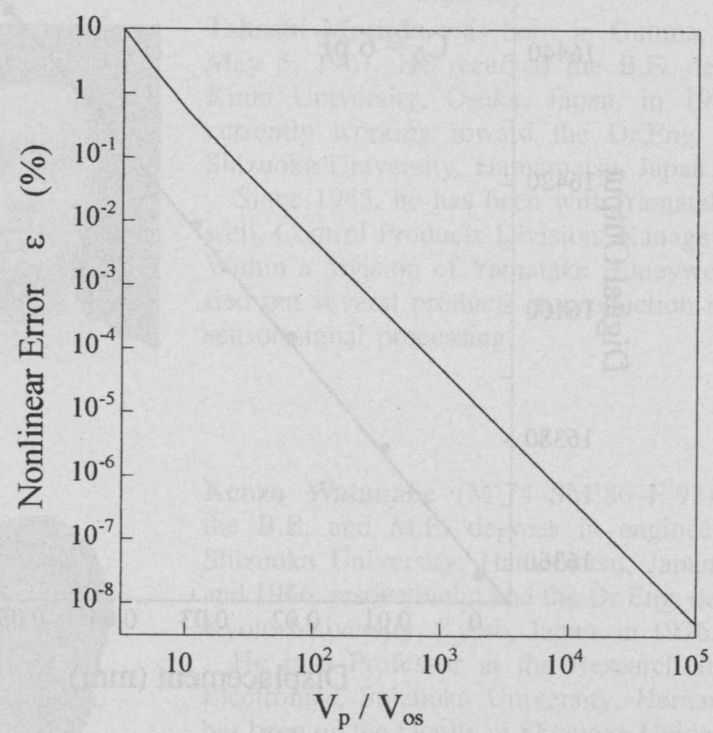

Fig. 5. Nonlinear error due to the offset voltage of opamps.

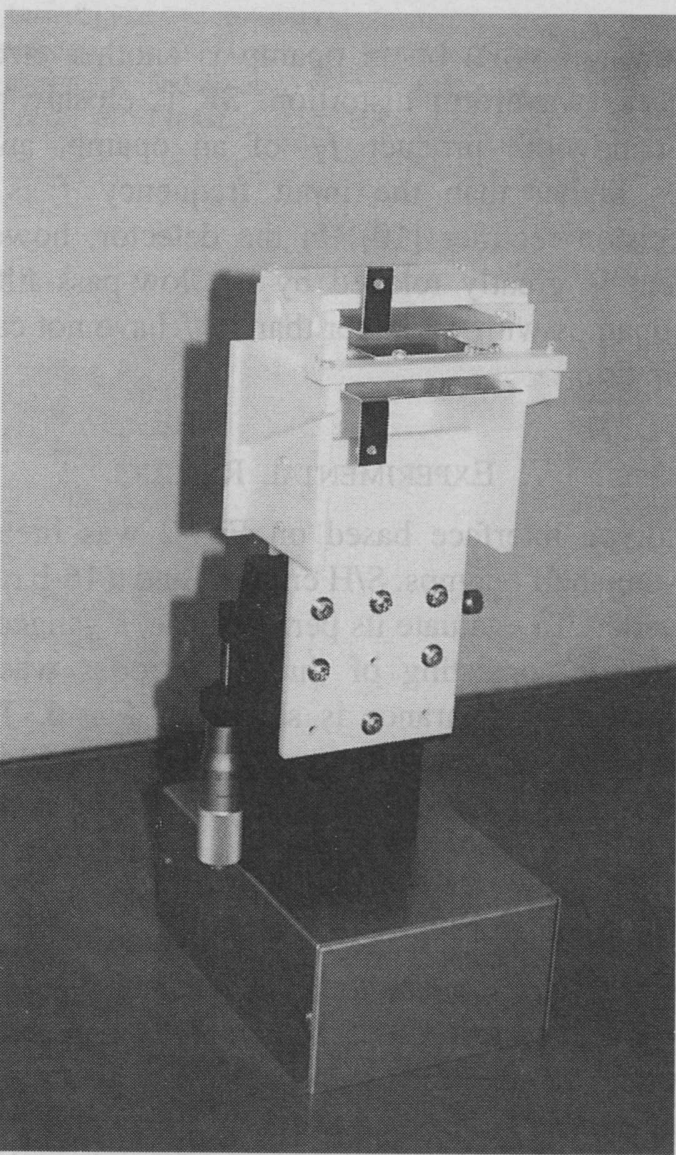

Fig. 6. A ganged parallel-plate capacitor used for experiments.

wiring, respectively. It indicates, therefore, that an accuracy better than $13 \mathrm{~b}$ is easily achievable without any special techniques and components.

A main error source in the detector is the offset voltage $V_{o s}$ of opamp which modulates the conduction period of diode $D_{1}$ in Fig. 3. Its effect on the linear detection can be evaluated by integrating the input sinusoidal signal over the conduction period. The results are shown in Fig. 5. Decreasing with the peak amplitude $V_{p}$ of the input signal, the nonlinear error becomes negligibly small if $V_{p}$ is 100 times larger than $V_{o s}$. 


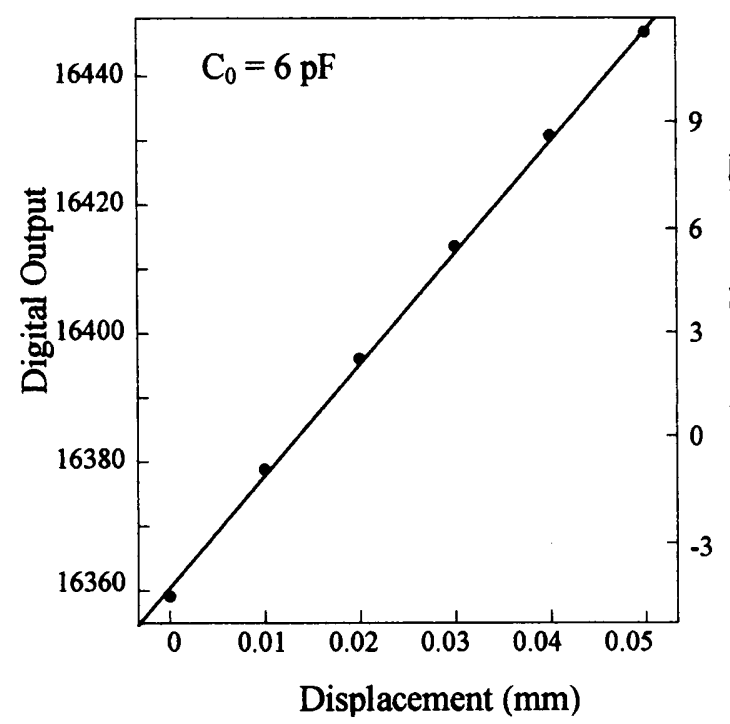

(a)

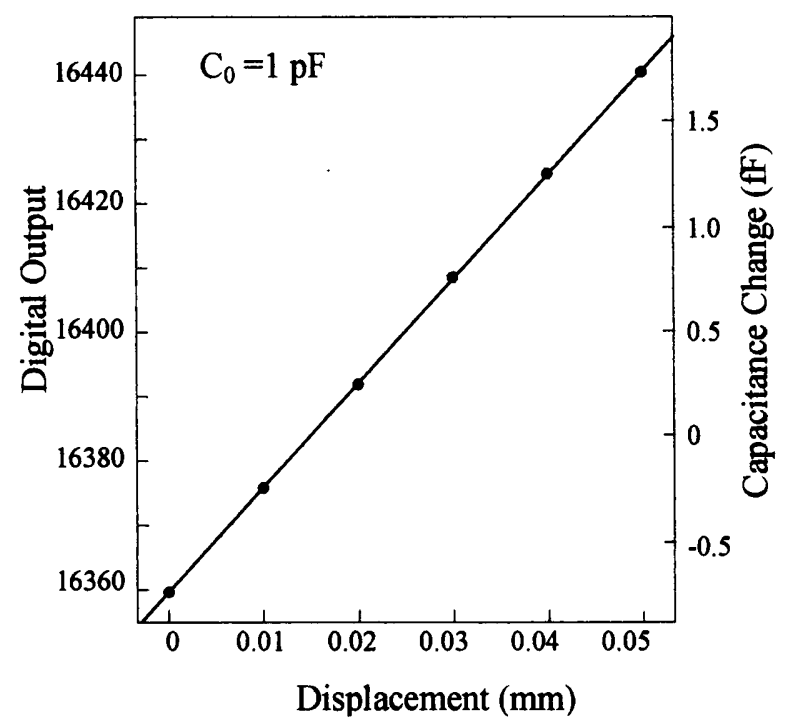

(b)

Fig. 7. Experimentally measured capacitance changes and the digital readings of the displacement when $C_{0}$ is (a) 6 and (b) 1 pF.

The slew rate (SR) of an opamp is another error source which causes waveform distortion. SR is closely related to the gain-bandwidth product $f_{T}$ of an opamp, and an $f_{T}$ 100 times higher than the input frequency $f$ is required for a precision rectifier [10]. In the detector, however, this requirement is greatly relaxed by the low-pass filtering. In practice, opamps with $f_{T}$ larger than $30 f$ have not caused any significant error.

\section{EXPERIMENTAL RESULTS}

A prototype interface based on Fig. 2 was breadboarded using off-the-shelf opamps, S/H circuits, and a 16-b ratiometric A/D converter. To evaluate its performance, a ganged parallelplate capacitor consisting of three electrodes was used as a transducer. Its appearance is shown in Fig. 6. Two outer electrodes are fixed while the inner electrode is driven by a micrometer screw to move back and forth. The stroke of the micrometer is $22 \mathrm{~mm}$, and its readings are accurate to $10 \mu \mathrm{m}$. The capacitances $C_{1}$ and $C_{2}$ are given by (3) and (4) with $x$ being the displacement of the inner electrode from the center of two outer electrodes. The total capacitance $C_{0}$ is $6 \mathrm{pF}$ when the electrodes measuring $47.5 \mathrm{~mm} \times 47.5 \mathrm{~mm}$ are used and is $1 \mathrm{pF}$ when the electrode dimensions are $10 \mathrm{~mm} \times 5 \mathrm{~mm}$. A guard electrode is installed to prevent the capacitance from being affected by surroundings. A $1-\mathrm{MHz}$ sinusoidal signal with $1 V_{p-p}$ amplitude is applied to the capacitor. Component values of the detector and the $\mathrm{S} / \mathrm{H}$ circuits are selected such that $V_{o 1}$ applied to the $\mathrm{A} / \mathrm{D}$ converter as the reference voltage is close to the recommended value of $3 \mathrm{~V}$, and the full-scale digital output is 32768 . The nonlinear error of the detector is less than $0.02 \%$.

Fig. 7 shows typical measurement results. Capacitance changes $\Delta C=C_{1}-C_{2}$, and the digital readings $\Delta C / C_{0}$ for displacement $x$ are indicated. Dots shown in the figures are average values of ten measurements. The standard deviation $\sigma$ was $0.21 \mathrm{fF}$ when $C_{0}=6 \mathrm{pF}$ and was $0.044 \mathrm{fF}$ when $C_{0}=1 \mathrm{pF}$. The maximum nonlinear error evaluated from the digital readings was $0.19 \mathrm{fF}$ when $C_{0}=6 \mathrm{pF}$ and was
$0.036 \mathrm{fF}$ when $C_{0}=1 \mathrm{pF}$. These data indicate that the measurement uncertainties decrease in proportion to $C_{0}$. As can be seen from Fig. 7, the capacitance change is $3 \mathrm{fF} / 10$ $\mu \mathrm{m}$ when $C_{0}=6 \mathrm{pF}$ and is $0.5 \mathrm{fF} / 10 \mu \mathrm{m}$ when $C_{0}=1 \mathrm{pF}$. The minimum scale of the micrometer is $10 \mu \mathrm{m}$. Therefore, it may be reasonably concluded that the measurement accuracy is wholly dominated by reading errors and backlash of the micrometer screw and that the signal processing accuracy of the interface is far beyond these limits.

Similar measurements were also repeated for a wide variety of opamps. No remarkable difference could be found for highspeed opamps with $f_{T}$ larger than $30 \mathrm{MHz}$. Excellent linearity was also found over a wide range of displacement. These preliminary measurements confirm the principles of operation and the accuracy estimate of the interface and prove its validity for high-accuracy signal processing of differential-capacitive transducers.

\section{CONCLUSIONS}

An interface circuitry of differential-capacitance transducer which performed the ratiometric operation of dividing one of the transducer capacitances by its total capacitance was described. The front end consisting of a $\mathrm{C} / \mathrm{V}$ converter and a detector was commonly used for the capacitance detection. This time-multiplexing capacitance detection using the common stage followed by the ratiometric operation using an $\mathrm{A} / \mathrm{D}$ converter is quite useful for high-accuracy signal processing. Analyses have shown that capacitance change as small as a few tenths of $\mathrm{fF}$ can be detected by this architecture. Performance evaluation of a prototype interface using a ganged parallel-plate capacitor has confirmed the accuracy estimate.

The interface described herein allows high-accuracy signal processing with standard components. The sampling speed higher than $5 \mathrm{ksps}$ can also be expected if a second-order low-pass filter is incorporated in the detector. These distinct features encourage its integrated circuit (IC) realization. Applications to practical transducers are another future work. 


\section{REFERENCES}

[1] T. Saigusa and S. Gotoh, "UNI $\Delta$ series electronic differential pressure transducer," Yokogawa Tech. J., vol. 22, pp. 23-29, Mar. 1978 (in Japanese).

[2] T. N. Toth and G. C. M. Meijer, "A low-cost smart capacitive position sensor,” IEEE Trans. Instrum. Meas., vol. 41, pp. 1041-1044, Dec. 1992.

[3] H. Matsumoto, H. Shimizu, and K. Watanabe, "A switched-capacitor charge-balancing analog-to-digital converter and its application to capacitance measurement," IEEE Trans. Instrum. Meas., vol. IM-36, pp. 873-878, Dec. 1987.

[4] Y. Cao and G. C. Temes, "High-accuracy circuit for on-chip capacitance ratio testing or sensor readout," IEEE Trans. Circuits Syst., vol. 41, pp. 637-639, Sept. 1994.

[5] T. N. Toth, G. C. M. Meijer, and H. M. M. Kerkvliet, "Ultra-linear, low-cost measurement system for multi-electrode pF-range capacitors," in Instrum. Meas. Tech. Conf. Proc., 1995, pp. 512-515.

[6] X. Li, G. C. M. Meijer, and G. W. de Jong, "An accurate smart capacitive angular-position sensor with a full circle range," in Instrum. Meas. Tech. Conf. Proc., 1995, pp. 80-83.

[7] R. F. Wolffenbuttel and P. P. L. Regtien, "Capacitance-to-phase angle conversion for the detection of extremely small capacities," IEEE Trans. Instrum. Meas., vol. IM-36, pp. 868-872, Dec. 1987.

[8] S. Ogawa, private communication.

[9] A. J. Peyton and V. Walsh, Analog Electronics with Op Amps. New York: Cambridge Univ. Press, 1993, ch. 11.

[10] K. Hayatleh, F. J. Lidgey, and S. Porta, "Degradation mechanism in operational amplifier precision rectifiers," IEEE Trans. Circuits Syst., vol. 42, pp. 479-485, Aug. 1995.

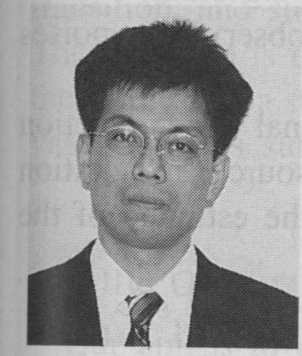

Kouji Mochizuki (M'98) was born in Shizuoka, Japan, on February 13, 1961. He received the B.E. and M.E. degrees in electronics engineering from Shizuoka University, Hamamatsu, Japan, in 1983 and 1985 , respectively.

From 1985 to 1989 , he was with Fujitsu Laboratories Ltd., where he worked on epitaxial growth of a III-V compound semiconductor. In July 1989, he joined Numazu College of Technology, Numazu, Japan, where he is mainly involved in resistance and capacitance measurements.

Mr. Mochizuki is a Member of the Institute of Electronics, Information and Communication Engineers of Japan.

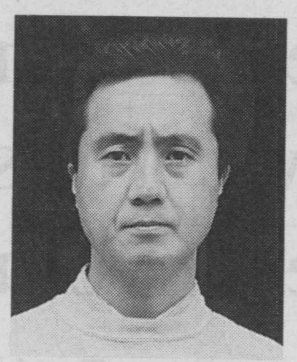

Takashi Masuda was born in Gunma, Japan, on May 5, 1961. He received the B.E. degree from Kinki University, Osaka, Japan, in 1985. He is currently working toward the Dr.Eng. degree at Shizuoka University, Hamamatsu, Japan.

Since 1985, he has been with Yamatake Honeywell, Control Products Division, Kanagawa, Japan Within a division of Yamatake Honeywell, he carried out several products in production sensor and sensor signal processing.

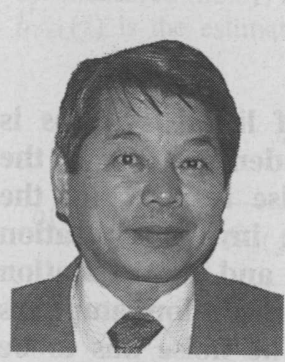

Kenzo Watanabe (M'74-SM'86-F'93) received the B.E. and M.E. degrees in engineering from Shizuoka University, Hamamatsu, Japan, in 1962 and 1966, respectively, and the Dr.Eng. degree from Kyoto University, Kyoto, Japan, in 1976.

$\mathrm{He}$ is a Professor at the Research Institute of Electronics, Shizuoka University, Hamamatsu. He has been on the faculty at Shizuoka University since 1962, serving progressively as a Research Assistant, Associate Professor, and Professor, except for a year as a Visiting Professor at the University of

California, Los Angeles.

Dr. Watanabe serves as an AdCom Member of the Instrumentation and Measurement Society and is an Associate Editor of the TRANSACTIONS ON InStRUMENTATION AND MEASUREMENT. He is a Member of the IMTC Board of Directors. 